\title{
Complete Invariance Property with respect to Homeomorphism over Frame Multiwavelet and Super-Wavelet Spaces
}

\author{
Saurabh Chandra Maury \\ Department of Mathematics, University of Allahabad, Allahabad 211 002, India \\ Correspondence should be addressed to Saurabh Chandra Maury; smaury94@gmail.com
}

Received 14 May 2014; Accepted 16 July 2014; Published 4 August 2014

Academic Editor: Ding-Xuan Zhou

Copyright (C) 2014 Saurabh Chandra Maury. This is an open access article distributed under the Creative Commons Attribution License, which permits unrestricted use, distribution, and reproduction in any medium, provided the original work is properly cited.

We discuss the complete invariance property with respect to homeomorphism (CIPH) over various sets of wavelets containing all orthonormal multiwavelets, all tight frame multiwavelets, all super-wavelets of length $n$, and all normalized tight super frame wavelets of length $n$.

\section{Introduction}

A topological space $X$ is said to possess the complete invariance property (CIP) if each of its nonempty closed sets is the fixed point set, for some continuous self-map $f$ on $X$ [1]. In case $f$ can be chosen to be a homeomorphism, the space is said to possess the complete invariance property with respect to homeomorphism (CIPH) [2]. These notions have been extensively studied by Schirmer, Martin, Nadler, Oversteegen, Tymchatyn, Weiss, Chigogidge, and Hofmann. They studied the preservation of these properties under various topological operations such as products, cones and wedge products. They obtained various spaces with or without these properties.

Recently, Dubey and Vyas in [3] have studied the topological notion of the complete invariance property over the set $\mathscr{W}$, of all one-dimensional orthonormal wavelets on $\mathbb{R}$ and certain subsets of $\mathscr{W}$. They noticed a free action of the unit circle $S^{1}$ on $\mathscr{W}$ and obtained each orbit isometric to $S^{1}$. They proved that the set of all one-dimensional orthonormal wavelets, the set of all MRA wavelets, and the set of all MSF wavelets on $\mathbb{R}$ have the complete invariance property with respect to homeomorphism employing the following result of Martin [2]: "A space X has the CIPH if it satisfies the following conditions: (i) $S^{1}$ acts on $X$ freely. (ii) $X$ possesses a bounded metric such that each orbit is isometric to $S^{1}$."

In this paper, we study the complete invariance property with respect to homeomorphism over the spaces $W \subset \prod_{1 \leq j \leq L} L^{2}\left(\mathbb{R}^{n}\right)$, containing all orthonormal multiwavelets on $\mathbb{R}^{n}$ in $L$-tuple form, $W_{T} \subset \prod_{1 \leq j \leq L} L^{2}\left(\mathbb{R}^{n}\right)$, containing all tight frame multiwavelets on $\mathbb{R}^{n}$ in $L$-tuple form, $\delta \mathscr{W}_{n}=\left\{\left(\eta_{1}, \ldots, \eta_{n}\right):\left(\eta_{1}, \ldots, \eta_{n}\right)\right.$ is a superwavelet of length $n$ for $\left.L^{2}(\mathbb{R})^{\oplus n}\right\}$, and $\mathcal{S} \mathscr{W}_{n}^{\mathrm{NT}}=\left\{\left(\eta_{1}, \ldots, \eta_{n}\right)\right.$ : $\left(\eta_{1}, \ldots, \eta_{n}\right)$ is a normalized tight super frame wavelet of length $n$ for $L^{2}(\mathbb{R})^{\oplus n}$. In case of the action of $S^{1}$ over $W, W_{T}$, and $\delta \mathscr{W}_{n}^{\mathrm{NT}}$ we obtain that the action is free but orbits are not isometric to $S^{1}$. Observing this fact, we have proved that the result of Martin stated above is also true for orbits isometric to a circle of finite radius.

\section{Prerequisites}

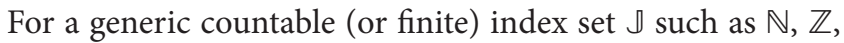
$\mathbb{N} \cup \mathbb{N}$, and $\mathbb{Z} \times \mathbb{Z}$, a collection of elements $\Phi=\left\{\phi_{j}: j \in \mathbb{J}\right\}$ in a separable Hilbert space $H$ is called a frame if there exist constants $A$ and $B, 0<A \leq B<\infty$, such that

$$
A\|f\|^{2} \leq \sum_{j \in \rrbracket}\left|\left\langle f, \phi_{j}\right\rangle\right|^{2} \leq B\|f\|^{2}, \quad \forall f \in H .
$$

The optimal constants (maximal for $A$ and minimal for $B$ ) are called the frame bounds. $A$ is called a lower frame bound and $B$ is called an upper frame bound of the frame. The frame $\left\{\phi_{j}: j \in \mathbb{J}\right\}$ is called a tight frame if $A=B$ and is called normalized tight frame if $A=B=1$. Any orthonormal basis 
in a Hilbert space is a normalized tight frame. Notice that, for a nonzero element $\phi_{k}$ of a frame $\Phi$ in $\mathbb{H}$, the following inequality holds:

$$
\left\|\phi_{k}\right\| \leq \sqrt{B}, \quad \forall k \in \mathbb{J} .
$$

This follows by noting that

$$
\left\|\phi_{k}\right\|^{4}=\left|\left\langle\phi_{k}, \phi_{k}\right\rangle\right|^{2} \leq \sum_{j \in \rrbracket}\left|\left\langle\phi_{k}, \phi_{j}\right\rangle\right|^{2} \leq B\left\|\phi_{k}\right\|^{2} .
$$

This shows that the elements of a frame need not be normal but they must have an upper bound.

Definition 1 (see [4]). Let $A$ be an $n \times n$ expansive matrix such that $A \mathbb{Z}^{n} \subset \mathbb{Z}^{n}$. Then a finite set $\Psi=\left\{\psi^{1}, \ldots, \psi^{L}\right\} \subset L^{2}\left(\mathbb{R}^{n}\right)$ is called an orthonormal multiwavelet if the collection $\mathscr{A}(\Psi)=$ $\left\{\psi_{j, k}^{l}: j \in \mathbb{Z}, k \in \mathbb{Z}^{n}, l=1, \ldots, L\right\}$ is an orthonormal basis for $L^{2}\left(\mathbb{R}^{n}\right)$, where for $\psi \in L^{2}\left(\mathbb{R}^{n}\right)$ one uses the convention

$$
\psi_{j, k}=|\operatorname{det} A|^{j / 2} \psi\left(A^{j} \cdot-k\right) .
$$

If a multiwavelet $\Psi$ consists of a single element $\psi$, then we say that $\psi$ is a wavelet. By an expansive matrix $A$, we mean a square matrix the moduli of whose eigenvalues are greater than 1 .

If the collection $\mathscr{A}(\Psi)=\left\{\psi_{j, k}^{l}: j \in \mathbb{Z}, k \in \mathbb{Z}^{n}, l=\right.$ $1, \ldots, L\}$ is a normalized tight frame, then the set $\Psi=$ $\left\{\psi^{1}, \ldots, \psi^{L}\right\} \subset L^{2}\left(\mathbb{R}^{n}\right)$ is called a normalized tight frame multiwavelet. Similarly, $\Psi$ is called a tight frame multiwavelet when the above collection $\mathscr{A}(\Psi)$ is a tight frame and a frame multiwavelet when the above collection $\mathscr{A}(\Psi)$ is a frame.

The following result establishes a characterization of normalized tight frame multiwavelet.

Theorem 2 (see [5]). Suppose $\Psi=\left\{\psi^{1}, \ldots, \psi^{L}\right\} \subset L^{2}\left(\mathbb{R}^{n}\right)$. Then the collection $\mathscr{A}(\Psi)=\left\{\psi_{j, k}^{l}: j \in \mathbb{Z}, k \in \mathbb{Z}^{n}, l=\right.$ $1, \ldots, L\}$ with a dilation $A$ is a normalized tight frame if and only if

(i) $\sum_{l=1}^{L} \sum_{j \in \mathbb{Z}}\left|\widehat{\psi}^{l}\left(B^{j} \xi\right)\right|^{2}=1$, for a.e. $\xi \in \mathbb{R}^{n}$, where $B$ is the transpose of $A$,

(ii) $t_{q}(\xi) \equiv \sum_{l=1}^{L} \sum_{j \geq 0} \widehat{\psi}^{l}\left(B^{j} \xi\right) \overline{\widehat{\psi}^{l}\left(B^{j}(\xi+q)\right)}=0$, for $q \epsilon$ $\mathbb{Z}^{n} \backslash B \mathbb{Z}^{n}$ and a.e. $\xi \in \mathbb{R}^{n}$.

In particular $\Psi$ is a multiwavelet if and only if the above conditions hold and $\left\|\psi^{l}\right\|=1$ for all $l=1, \ldots, L$. by

The Fourier transform of $f \in L^{1}\left(\mathbb{R}^{n}\right) \cap L^{2}\left(\mathbb{R}^{n}\right)$ is defined

$$
\widehat{f}(\xi)=\int_{\mathbb{R}^{n}} f(x) e^{-2 \pi i\langle\xi, x\rangle} d x, \quad \xi \in \mathbb{R}^{n},
$$

where $\langle\xi, x\rangle$ denotes the real inner product.

Since $L^{1}\left(\mathbb{R}^{n}\right) \cap L^{2}\left(\mathbb{R}^{n}\right)$ is a dense subset of $L^{2}\left(\mathbb{R}^{n}\right)$, this definition extends uniquely to $L^{2}\left(\mathbb{R}^{n}\right)$.

One of the methods of constructing orthonormal wavelets is based on multiresolution analysis which is a family of closed subspaces of $L^{2}\left(\mathbb{R}^{n}\right)$ satisfying certain properties.

Let $\Psi=\left\{\psi^{1}, \ldots, \psi^{L}\right\} \subset L^{2}\left(\mathbb{R}^{n}\right)$ be an orthonormal multiwavelet associated with a dilation $A$.

Then

$$
D_{\Psi}(\xi)=\sum_{l=1}^{L} \sum_{j \in \mathbb{N}} \sum_{k \in \mathbb{Z}^{n}}\left|\widehat{\psi}^{l}\left(B^{j}(\xi+k)\right)\right|^{2}, \quad \text { for a.e. } \xi \in \mathbb{R}^{n},
$$

describes the dimension function $D_{\Psi}$ for $\Psi$, where $B$ is the transpose of $A$.

We have the following result analogous to that as in the case of one dimension.

Theorem 3 (see [6]). An orthonormal multiwavelet $\Psi \in$ $L^{2}\left(\mathbb{R}^{n}\right)$ is an MRA multiwavelet if and only if $D_{\Psi}(\xi)=1$, for a.e. $\xi \in \mathbb{R}^{n}$.

Definition 4 (see [6]). An MSF (minimally supported frequency) multiwavelet (of order $L$ ) is an orthonormal multiwavelet $\Psi=\left\{\psi^{1}, \ldots, \psi^{L}\right\}$ such that $\left|\widehat{\psi}^{l}\right|=\chi_{W_{l}}$ for some measurable set $W_{l} \subset \mathbb{R}^{n}, l=1, \ldots, L$. An MSF multiwavelet of order 1 is simply referred to as an MSF wavelet.

The following theorem characterizes all MSF multiwavelets.

Theorem 5 (see [7]). A set $\Psi=\left\{\psi^{1}, \ldots, \psi^{L}\right\} \subset L^{2}\left(\mathbb{R}^{n}\right)$ such that $\left|\widehat{\psi}^{l}\right|=\chi_{W_{l}}$ for $l=1, \ldots, L$ is an orthonormal multiwavelet with the dilation matrix $A$ if and only if

$$
\begin{gathered}
\sum_{k \in \mathbb{Z}^{n}} \chi_{W_{l}}(\xi+k) \chi_{W_{l^{\prime}}}(\xi+k)=\delta_{l, l^{\prime}} \\
\text { a.e. } \xi \in \mathbb{R}^{n}, \quad l, l^{\prime}=i, \ldots, L, \\
\sum_{l=1}^{L} \sum_{j \in \mathbb{Z}} \chi_{W_{l}}\left(B^{j} \xi\right)=1, \quad \text { a.e., } \xi \in \mathbb{R}^{n}, B=A^{T} .
\end{gathered}
$$

In [8], Han and Larson have introduced the notion of super-wavelet which has applications in signal processing, data compression, and image analysis.

Definition 6. Suppose that $\eta_{1}, \eta_{2}, \ldots, \eta_{n}$ are normalized tight frame wavelets for $L^{2}(\mathbb{R})$. One will call the $n$-tuple $\left(\eta_{1}, \ldots, \eta_{n}\right)$ a super-wavelet of length $n$ if

$$
\left\{D^{j} T^{k} \eta_{1} \oplus \cdots \oplus D^{j} T^{k} \eta_{n}: j, k \in \mathbb{Z}\right\}
$$

is an orthonormal basis for $L^{2}(\mathbb{R}) \oplus \cdots \oplus L^{2}(\mathbb{R})\left(\right.$ say, $\left.L^{2}(\mathbb{R})^{\oplus n}\right)$, where

$$
\begin{gathered}
D^{j} f(x)=2^{j / 2} f\left(2^{j} x\right), \\
T^{k} f(x)=f(x-k) \quad \text { for } f \in L^{2}(\mathbb{R}) .
\end{gathered}
$$

Han and Larson in their memoirs [8] proved that, for each $n$ ( $n$ can be $\infty$ ), there is a super-wavelet of length $n$.

Following is a characterization of a super-wavelet of length $n$. 
Theorem 7. Let $\eta_{1}, \ldots, \eta_{n} \in L^{2}(\mathbb{R})$. Then $\left(\eta_{1}, \ldots, \eta_{n}\right)$ is a super-wavelet of length $n$ if and only if the following equations hold:

(i) $\sum_{j \in \mathbb{Z}}\left|\widehat{\eta}_{i}\left(2^{j} \xi\right)\right|^{2}=1$, for a.e. $\xi \in \mathbb{R}, i=1, \ldots, n$,

(ii) $\sum_{j=0}^{\infty} \widehat{\eta}_{i}\left(2^{j} \xi\right) \overline{\widehat{\eta}_{i}\left(2^{j}(\xi+k)\right)}=0$, for a.e. $\xi \in \mathbb{R}, k \in 2 \mathbb{Z}+$ $1, i=1, \ldots, n$,

(iii) $\sum_{j \in \mathbb{Z}} \sum_{i=1}^{n}\left|\widehat{\eta}_{i}(\xi+k)\right|^{2}=1$, for a.e. $\xi \in \mathbb{R}$,

(iv) $\sum_{\mathbb{N} \text {. }}^{\infty} \sum_{i=1}^{m} \widehat{\eta}_{i}\left(2^{j}(\xi+k)\right) \overline{\widehat{\eta}_{i}(\xi+k)}=0$, for a.e. $\xi \in \mathbb{R}, j \in$

Definition 8. A super-wavelet $\left(\eta_{1}, \ldots, \eta_{n}\right)$ is said to be an MRA super-wavelet if every $\eta_{i}(i=1, \ldots, n)$ is an MRA frame wavelet.

Definition 9(see [9]). Suppose that $\left(\eta_{1}, \eta_{2}, \ldots, \eta_{n}\right) \in L^{2}(\mathbb{R})^{\oplus n}$. One will call the $n$-tuple $\left(\eta_{1}, \ldots, \eta_{n}\right)$ a normalized tight super frame wavelet of length $n$ if

$$
\left\{D^{j} T^{k} \eta_{1} \oplus \cdots \oplus D^{j} T^{k} \eta_{n}: j, k \in \mathbb{Z}\right\}
$$

is a normalized tight frame for $L^{2}(\mathbb{R})^{\oplus n}$.

For a self-continuous map $f$ on a topological space $X$, Fix $f$ denotes the set of all fixed points of $f$. A point $x \in X$ is called a fixed point of $f$ if $f(x)=x$. In case $X$ is Hausdorff, Fix $f$ is a closed set.

From Brouwer's fixed point theorem, it follows that Fix $f$ for a self-continuous map $f$ on the disc is a nonempty closed set. The converse of this result was considered by Robbins who found it to be true [10]. This is what led to the notion of the complete invariance property. Formally, we have the following.

Definition 10 (see [1]). A topological space $X$ is said to possess the complete invariance property (CIP) if each of its nonempty closed sets is $\operatorname{Fix} f$, for some self-continuous map $f$ on $X$.

Definition 11 (see [2]). A topological space $X$ is said to possess the complete invariance property with respect to homeomorphism (CIPH) if each of its nonempty closed sets is the fixed point set, $\operatorname{Fix} f$, for some self-homeomorphism $f$ on $X$.

Theorem 12 (see [2]). A space X has the CIPH if it satisfies the following conditions:

(i) $S^{1}$ acts on $X$ freely;

(ii) $X$ possesses a bounded metric such that each orbit is isometric to the unit circle.

\section{Frame Multiwavelet Spaces and the CIPH}

From Theorem 12 it is clear that to examine the CIPH over a metric space $X$ we need a free $S^{1}$ action on $X$ having orbits isometric to the unit circle. This result does not provide any information about the CIPH over $X$ in case the radii of orbits are different from unity.
Below we modify the above result and show that if $S^{1}$ acts freely on a metric space $X$ and orbits are isometric to circles of finite radii, then $X$ has the CIPH.

Theorem 13. A space $X$ has the CIPH if it satisfies the following conditions:

(i) $S^{1}$ acts on $X$ freely;

(ii) $X$ possesses a bounded metric such that each orbit is isometric to $S_{r}^{1}$, a circle of radius $r$, where $0<r \leq L$ for some $L>0$.

Proof. Let $(X, d)$ be a metric space with $d \leq 3 \pi L$ and let $*$ : $X \times S^{1} \rightarrow X$ be the action satisfying conditions (i) and (ii). For a nonempty closed set $A$ in $X$ set $a(x)=(1 / 2 L) d(x, A)$ and define $f: X \rightarrow X$ by

$$
f(x)=x * e^{i a(x)}, \quad x \in X .
$$

Since $0<a(x)<2 \pi$ if $x \notin A$, it follows that Fix $f=A$. To see that $f$ is one-one suppose $f(y)=f(z)$. Then $y$ and $z$ must lie on the same orbit isometric to the circle $S_{r}^{1}, 0<r \leq L$, and, for some real number $t, y=z * e^{i t}$ with $d(y, z)=|r t| \leq \pi L$.

Thus $y * e^{i a(y)}=z * e^{i a(z)}$ and hence, for some integer $n$,

$$
t+a(y)-a(z)=2 \pi n .
$$

By the triangle inequality applying over $y, z, A$ we have

$$
2 L|a(y)-a(z)| \leq|r t| \leq \pi L,
$$

and so

$$
|a(y)-a(z)| \leq \frac{\pi}{2}
$$

Thus the equation $t+a(y)-a(z)=2 \pi n$ holds only for $n=0$ and hence $t=0$.

Since $f$ is an orbit wise one-one map and a homeomorphism of $S_{r}^{1}$ into itself must be onto, it follows that $f$ is onto. In order to conclude that $f$ is a homeomorphism it suffices to show that $f$ is a closed mapping. For the remaining portion see the proof of Theorem 2.2 [2].

Let $A$ be an expansive matrix and $L \geq 1$ is an integer. Then the space

$$
\begin{aligned}
& W_{\mathrm{NT}}=\left\{\left(\psi^{1}, \ldots, \psi^{L}\right) \in \prod_{1 \leq j \leq L} L^{2}\left(\mathbb{R}^{n}\right):\left\{\psi^{1}, \ldots, \psi^{L}\right\}\right. \\
&\text { is a normalized tight frame multiwavelet }\}
\end{aligned}
$$

is a metric space with the natural metric $d$ defined by

$$
d^{2}(\Psi, \Phi)=\sum_{l=1}^{L}\left\|\psi^{l}-\phi^{l}\right\|_{2}^{2},
$$

where $\Psi=\left(\psi^{1}, \ldots, \psi^{L}\right)$ and $\Phi=\left(\phi^{1}, \ldots, \phi^{L}\right)$. 
Theorem 14. The space $W_{N T}$ has the CIPH.

Proof. The characterization of a normalized tight frame multiwavelet (Theorem 2) provides that if $e^{i \theta} \in S^{1}$ and $\Psi=$ $\left(\psi^{1}, \ldots, \psi^{L}\right) \in W_{\mathrm{NT}}$, then, $e^{i \theta} \cdot \Psi=\left(e^{i \theta} \cdot \psi^{1}, \ldots, e^{i \theta} \cdot \psi^{L}\right) \in W_{\mathrm{NT}}$. For $\Psi \in W_{\mathrm{NT}}$ the collection $\mathscr{A}(\Psi)=\left\{\psi_{j, k}^{l}: j \in \mathbb{Z}, k \in\right.$ $\left.\mathbb{Z}^{n}, l=1, \ldots, L\right\}$ forms a normalized tight frame for $L^{2}\left(\mathbb{R}^{n}\right)$. This shows that $\Psi$ cannot be a zero function in $L^{2}\left(\mathbb{R}^{n}\right)$. Thus, the function $\eta: S^{1} \times W_{\mathrm{NT}} \rightarrow W_{\mathrm{NT}}$ defined by

$$
\eta\left(e^{i \theta}, \Psi\right)=e^{i \theta} \cdot \Psi, \quad e^{i \theta} \in S^{1}, \Psi \in W_{\mathrm{NT}}
$$

is a free action of $S^{1}$ on $W_{\mathrm{NT}}$.

For the continuity of $\eta$ at $\left(e^{i \theta}, \Psi\right)$, we simply observe that

$$
\begin{aligned}
\left\|\eta\left(e^{i \theta}, \Psi\right)-\eta\left(e^{i \theta_{1}}, \Psi_{1}\right)\right\| & \leq\left|e^{i \theta}-e^{i \theta_{1}}\right|\|\Psi\|+\left\|\Psi-\Psi_{1}\right\| \\
& \leq\left|e^{i \theta}-e^{i \theta_{1}}\right| \sqrt{L}+\left\|\Psi-\Psi_{1}\right\|,
\end{aligned}
$$

where $\left(e^{i \theta_{1}}, \Psi_{1}\right) \in S^{1} \times W_{\mathrm{NT}}$ and

$$
\|\Psi\| \leq \sqrt{\left\|\psi^{1}\right\|_{2}^{2}+\cdots+\left\|\psi^{L}\right\|_{2}^{2}} \leq \sqrt{L}
$$

The orbit of $\Psi$ is given by

$$
\eta\left(S^{1} \times\{\Psi\}\right)=\left\{\eta\left(e^{i \theta}, \Psi\right): \theta \in[0,2 \pi)\right\},
$$

which is isometric to $S_{r}^{1}$, the circle of radius $0<r \leq \sqrt{L}$, via the map

$$
\varphi: \eta\left(S^{1} \times\{\Psi\}\right) \longrightarrow S_{r}^{1}
$$

which sends $e^{i \theta} \cdot \Psi$ to $r e^{i \theta}$, where $r=\|\Psi\|$. Thus from Theorem 13 it follows that the space $W_{\mathrm{NT}}$ has the CIPH.

Corollary 15. If $A$ is an expansive matrix and $L \geq 1$ is an integer, then the space

$$
\begin{gathered}
W=\left\{\left(\psi^{1}, \ldots, \psi^{L}\right) \in \prod_{1 \leq j \leq L} L^{2}\left(\mathbb{R}^{n}\right):\left\{\psi^{1}, \ldots, \psi^{L}\right\}\right. \\
\quad \text { is an orthonormal multiwavelet }\}
\end{gathered}
$$

has the CIPH.

Proof. Note that $W \subset W_{\mathrm{NT}}$. The restriction $\eta / W$ of the action $\eta$ to $W$ is a free action. The orbit of $\Psi$ is isometric to $S_{\sqrt{L}}^{1}$, the circle of radius $\sqrt{L}$, via the map sending $e^{i \theta} \cdot \Psi$ to $\sqrt{L} e^{i \theta}$, where $\sqrt{L}=\|\Psi\|$. Thus from Theorem 13 it follows that the space $W$ has the CIPH.

Remark 16. Let $W^{M}=\left\{\left(\psi^{1}, \ldots, \psi^{L}\right) \in W:\left\{\psi^{1}, \ldots, \psi^{L}\right\}\right.$ is an MRA multiwavelet $\}$ and $W^{S}=\left\{\left(\psi^{1}, \ldots, \psi^{L}\right) \in\right.$ $W:\left\{\psi^{1}, \ldots, \psi^{L}\right\}$ is an MSF multiwavelet $\}$. By noting that the dimension function of $\Psi, D(\Psi)$, is equal to $D\left(\eta\left(e^{i \theta}, \Psi\right)\right)$, it follows from Theorem 3 that $\Psi$ is an MRA wavelet if and only if $\eta\left(e^{i \theta}, \Psi\right)$ is an MRA wavelet. Also, we note that $\Psi$ is an MSF wavelet if and only if $\eta\left(e^{i \theta}, \Psi\right)$ is an MSF wavelet. Thus $W^{M}$, $W^{S}$, and $W^{M} \cap W^{S}$ are invariant sets in $W$ with respect to the action of topological group $S^{1}$. The orbits of these invariant sets remain isometric to $S_{\sqrt{L}}^{1}$. Thus from Theorem 13 it follows that the spaces $W^{M}, W^{S}$, and $W^{M} \cap W^{S}$ have the CIPH.

In the case of tight frame, the frame bounds $A$ and $B$ are equal but need not be 1 . After a renormalization, we can assume $A=B=1$. If we denote

$$
\begin{gathered}
W_{T}=\left\{\left(\psi^{1}, \ldots, \psi^{L}\right) \in \prod_{1 \leq j \leq L} L^{2}\left(\mathbb{R}^{n}\right):\left\{\psi^{1}, \ldots, \psi^{L}\right\}\right. \\
\text { is a tight frame multiwavelet }\},
\end{gathered}
$$

then we have the following result.

Theorem 17. The space $W_{T}$ has the CIPH.

Theorem 18. Let

$$
\begin{aligned}
W_{B_{0}}=\{ & \left(\psi^{1}, \ldots, \psi^{L}\right) \in \prod_{1 \leq j \leq L} L^{2}\left(\mathbb{R}^{n}\right):\left\{\psi^{1}, \ldots, \psi^{L}\right\} \\
& \text { is a frame multiwavelet with the } \\
& \text { upper frame bound bounded by } \left.B_{0}\right\} .
\end{aligned}
$$

Then the space $W_{B_{0}}$ has the CIPH.

Proof. Let $\Psi=\left(\psi^{1}, \ldots, \psi^{L}\right) \in W_{B_{0}}$; that is, the collection

$$
\mathscr{A}(\Psi)=\left\{\Psi_{j, k}^{l}: j \in \mathbb{Z}, k \in \mathbb{Z}^{n} \text { and } l=1, \ldots, L\right\}
$$

is a frame of $L^{2}\left(\mathbb{R}^{n}\right)$

Then

$$
A\|f\|_{2}^{2} \leq \sum_{l=1}^{L} \sum_{j \in \mathbb{Z}} \sum_{k \in \mathbb{Z}^{n}}\left|\left\langle f, \psi_{j, k}^{l}\right\rangle\right|^{2} \leq B\|f\|_{2}^{2},
$$

for all $f \in L^{2}\left(\mathbb{R}^{n}\right)$, where $A$ and $B$ are frame bounds of the frame generated by $\Psi$.

Now, we show that $e^{i \theta} \cdot \Psi$ is an element of $W_{B_{0}}$. That is,

$$
A\|f\|_{2}^{2} \leq \sum_{l=1}^{L} \sum_{j \in \mathbb{Z}} \sum_{k \in \mathbb{Z}^{n}}\left|\left\langle f, e^{i \theta} \psi_{j, k}^{l}\right\rangle\right|^{2} \leq B\|f\|_{2}^{2},
$$

for all $f \in L^{2}\left(\mathbb{R}^{n}\right)$.

Note that

$$
\left\langle f, e^{i \theta} \psi_{j, k}^{l}\right\rangle=\int f \overline{e^{i \theta} \psi_{j, k}^{l}}=\overline{e^{i \theta}} \int f \overline{\psi_{j, k}^{l}} .
$$


Hence we have

$$
\left|\left\langle f, \psi_{j, k}^{l}\right\rangle\right|^{2}=\left|\left\langle f, e^{i \theta} \psi_{j, k}^{l}\right\rangle\right|^{2} .
$$

Thus the map

$$
\eta: S^{1} \times W_{B_{0}} \longrightarrow W_{B_{0}}
$$

defined by $\eta\left(e^{i \theta}, \Psi\right)=e^{i \theta} \cdot \Psi$ is well defined and describes a free action of $S^{1}$ on $W_{B_{0}}$.

The continuity of $\eta$ at $\left(e^{i \theta}, \Psi\right)$ follows by noting that

$$
\begin{aligned}
& \left\|\eta\left(e^{i \theta}, \Psi\right)-\eta\left(e^{i \theta_{1}}, \Psi_{1}\right)\right\| \\
& \quad \leq\left|e^{i \theta}-e^{i \theta_{1}}\right| \sqrt{L B_{0}}+\left\|\Psi-\Psi_{1}\right\|,
\end{aligned}
$$

where $\left(e^{i \theta_{1}}, \Psi_{1}\right) \in S^{1} \times W_{B_{0}}$ and

$$
\|\Psi\|=\sqrt{\left\|\psi^{1}\right\|_{2}^{2}+\cdots+\left\|\psi^{L}\right\|_{2}^{2}} \leq \sqrt{L B_{0}} .
$$

The orbit of $\Psi$ is isometric to $S_{r}^{1}$, the circle of radius $0<$ $r \leq \sqrt{L B_{0}}$, via the map sending $e^{i \theta} \cdot \Psi$ to $r e^{i \theta}$, where $r=\|\Psi\|$; hence, the result is obtained.

\section{Super-Wavelets and the CIPH}

The concept of super-wavelets was first introduced and studied in [8]. Due to its potential applications in multiplexing techniques such as time division multiple access and frequency division multiple access, super-wavelet has attracted the attentions of some mathematicians and engineering specialists. In this section we study the topological notion of the complete invariance property with respect to homeomorphism over the sets of super-wavelets and normalized tight super frame wavelets.

Theorem 19. Let $n \geq 2$ be an integer. Consider the set $\delta \mathscr{W}_{n}$ defined by

$$
\begin{aligned}
\mathcal{S} \mathscr{W}_{n}=\{ & \left\{=\left(\eta_{1}, \ldots, \eta_{n}\right):\left(\eta_{1}, \ldots, \eta_{n}\right)\right. \\
& \text { is a super-wavelet of length } \left.n \text { for } L^{2}(\mathbb{R})^{\oplus n}\right\} .
\end{aligned}
$$

Then the space $\delta \mathscr{W}_{n}$ has the CIPH.

Proof. Let $\mathcal{\vartheta}=\left(\eta_{1}, \ldots, \eta_{n}\right)$ be an element of $\delta \mathscr{W}_{n}$. From Theorem 7 it follows that $e^{i \theta} \cdot \mathcal{\vartheta}=\left(e^{i \theta} \eta_{1}, \ldots, e^{i \theta} \eta_{n}\right)$ remains in $\delta \mathscr{W}_{n}$, where $e^{i \theta} \in S^{1}$. Thus the map

$$
\eta: S^{1} \times \delta \mathscr{W}_{n} \longrightarrow \delta \mathscr{W}_{n}
$$

defined by

$$
\eta\left(e^{i \theta}, \vartheta\right)=e^{i \theta} \cdot \vartheta, \quad \vartheta \in \mathcal{S} \mathscr{W}_{n}, e^{i \theta} \in S^{1}
$$

is a free action.

The continuity of $\eta$ at $\left(e^{i \theta}, 9\right)$ follows by noting that

$$
\left\|\eta\left(e^{i \theta}, \vartheta\right)-\eta\left(e^{i \theta_{1}}, \vartheta_{1}\right)\right\| \leq\left|e^{i \theta}-e^{i \theta_{1}}\right|+\left\|\vartheta-\vartheta_{1}\right\|,
$$

where $\left(e^{i \theta_{1}}, \vartheta_{1}\right) \in S^{1} \times \mathcal{S} \mathscr{W}_{n}$ and $\|\vartheta\|=1$.
The orbit of $\vartheta$ is given by

$$
\eta\left(S^{1} \times\{\vartheta\}\right)=\left\{\eta\left(e^{i \theta}, \vartheta\right): \theta \in[0,2 \pi)\right\},
$$

which is isometric to $S^{1}$, via the map

$$
\varphi: \eta\left(S^{1} \times\{\vartheta\}\right) \longrightarrow S^{1},
$$

which sends $e^{i \theta} \cdot \vartheta$ to $e^{i \theta}$. Thus from Theorem 12 it follows that the space $\delta \mathscr{W}_{n}$ has the CIPH.

Remark 20. If $\mathcal{S} \mathscr{W}_{n}^{M}=\left\{\left(\eta_{1}, \ldots, \eta_{n}\right):\left(\eta_{1}, \ldots, \eta_{n}\right)\right.$ is an MRA super-wavelet of length $n$ for $\left.L^{2}(\mathbb{R})^{\oplus n}\right\}$, then, for each $\left(\eta_{1}, \ldots, \eta_{n}\right) \in \delta \mathscr{W}_{n}^{M},\left(e^{i \theta} \eta_{1}, \ldots, e^{i \theta} \eta_{n}\right)$ is also an MRA superwavelet. Thus $\delta \mathscr{W}_{n}^{M}$ is an invariant set with respect to the action of $S^{1}$. Orbits of these invariant sets are isometric to the unit circle. Thus from Theorem 12 it follows that the space $\delta \mathscr{W}_{n}^{M}$ has the CIPH.

Theorem 21. Let $n \geq 2$ be an integer. Consider the set $\delta \mathscr{W}_{n}^{N T}$ defined by $\delta \mathscr{W}_{n}^{N T}=\left\{\vartheta=\left(\eta_{1}, \ldots, \eta_{n}\right):\left(\eta_{1}, \ldots, \eta_{n}\right)\right.$ is a normalized tight super frame wavelet of length $n$ for $\left.L^{2}(\mathbb{R})^{\oplus n}\right\}$. Then the space $\delta \mathscr{W}_{n}^{N T}$ has the CIPH.

Proof. Let $\vartheta=\left(\eta_{1}, \ldots, \eta_{n}\right) \in \mathcal{S} \mathscr{W}_{n}^{\mathrm{NT}}$. For $f=\left(f_{1}, \ldots, f_{n}\right) \in$ $L^{2}(\mathbb{R})^{\oplus n}$ we have

$$
\begin{aligned}
\|f\|^{2} & =\sum_{j \in \mathbb{Z}} \sum_{k \in \mathbb{Z}}\left|\left\langle f, D^{j} T^{k} \eta_{1} \oplus \cdots \oplus D^{j} T^{k} \eta_{n}\right\rangle\right|^{2} \\
& =\sum_{j \in \mathbb{Z}} \sum_{k \in \mathbb{Z}}\left|\left\langle f_{1}, D^{j} T^{k} \eta_{1}\right\rangle+\cdots+\left\langle f_{n}, D^{j} T^{k} \eta_{n}\right\rangle\right|^{2} \\
& =\sum_{j \in \mathbb{Z}} \sum_{k \in \mathbb{Z}}\left|\left\langle f_{1}, D^{j} T^{k} e^{i \theta} \eta_{1}\right\rangle+\cdots+\left\langle f_{n}, D^{j} T^{k} e^{i \theta} \eta_{n}\right\rangle\right|^{2} .
\end{aligned}
$$

This shows that $e^{i \theta} \cdot \mathcal{\vartheta}=\left(e^{i \theta} \eta_{1}, \ldots, e^{i \theta} \eta_{n}\right)$ remains in $\delta \mathscr{W}_{n}^{\mathrm{NT}}$, where $e^{i \theta} \in S^{1}$.

Thus the map

$$
\eta: S^{1} \times \delta \mathscr{W}_{n}^{\mathrm{NT}} \longrightarrow \delta \mathscr{W}_{n}^{\mathrm{NT}}
$$

defined by

$$
\eta\left(e^{i \theta}, \vartheta\right)=e^{i \theta} \cdot \vartheta, \quad \vartheta \in \mathcal{S} \mathscr{W}_{n}^{\mathrm{NT}}, e^{i \theta} \in S^{1}
$$

is a free action.

The continuity of $\eta$ at $\left(e^{i \theta}, 9\right)$ follows by noting that

$$
\begin{aligned}
\left\|\eta\left(e^{i \theta}, \vartheta\right)-\eta\left(e^{i \theta_{1}}, \vartheta_{1}\right)\right\| & =\left\|e^{i \theta} \cdot \vartheta-e^{i \theta_{1}} \cdot \vartheta_{1}\right\| \\
& \leq\left|e^{i \theta}-e^{i \theta_{1}}\right|+\left\|\vartheta-\vartheta_{1}\right\|,
\end{aligned}
$$

where $\left(e^{i \theta_{1}}, \vartheta_{1}\right) \in S^{1} \times \mathcal{S} \mathscr{W}_{n}^{\mathrm{NT}}$ and $\|\vartheta\| \leq 1$.

The orbit of $\vartheta$ is isometric to $S_{r}^{1}, 0<r \leq 1$, where $r=\|\vartheta\|$. Thus from Theorem 13 it follows that the space $\delta \mathscr{W}_{n}^{\mathrm{NT}}$ has the $\mathrm{CIPH}$. 
For a $d \times d$, real expansive matrix $A$, let $D_{A}$ and $T^{k}\left(k \in \mathbb{R}^{d}\right)$ be the unitary operators on $L^{2}\left(\mathbb{R}^{d}\right)$ defined by

$$
\begin{aligned}
\left(D_{A} f\right)(x) & =|A|^{1 / 2} f(A x), \\
T^{k} f(x) & =f(x-k), \quad \text { for } f \in L^{2}\left(\mathbb{R}^{d}\right) .
\end{aligned}
$$

Then we have the following.

Definition 22 (see [9]). Suppose that $\eta_{1}, \ldots, \eta_{n}$ are $A$-dilation single normalized tight frame wavelets. One calls the $n$ tuple $\left(\eta_{1}, \ldots, \eta_{n}\right)$ an $A$-dilation normalized tight super frame wavelet of length $n$ if

$$
\left\{D_{A}^{j} T^{k} \eta_{1} \oplus \cdots \oplus D_{A}^{j} T^{k} \eta_{n}: j \in \mathbb{Z}, k \in \mathbb{Z}^{d}\right\}
$$

is an $A$-dilation normalized tight frame for $L_{2}\left(\mathbb{R}^{d}\right)^{\oplus n}$.

Thus from the above definition analogous result to Theorem 21 holds in case of higher dimension as well.

Remark 23. Consider the set

$$
\begin{aligned}
\mathcal{S}=\{ & \left\{W, W^{M}, W^{S}, W^{M} \cap W^{S},\right. \\
& \left.W_{\mathrm{NT}}, W_{T}, W_{B_{0}}, \delta \mathscr{W}_{n}, \delta \mathscr{W}_{n}^{M}, \delta \mathscr{W}_{n}^{\mathrm{NT}}\right\} .
\end{aligned}
$$

Then we have similar results to the one-dimensional orthonormal wavelet case provided in [3]. Let $X, Y \in \mathcal{S}$. Then the product space $X \times Y$, the cylinder $X \times I$, where $I$ is the unit closed interval of the real line, the cone $C(X)$, and the suspension $S(X)$ have the CIP.

\section{Conflict of Interests}

The author declares that there is no conflict of interests regarding the publication of this paper.

\section{Acknowledgments}

The author would like to thank Dr. Niraj K. Shukla, Discipline of Mathematics, Indian Institute of Technology Indore, Indore, India, for his help and suggestion in wavelet theory and the referee for his (her) careful reading of the paper and suggestions. This work was supported by CSIR grant, New Delhi, India.

\section{References}

[1] L. E. Ward Jr., "Fixed point sets," Pacific Journal of Mathematics, vol. 47, pp. 553-565, 1973.

[2] J. R. Martin, "Fixed point sets of homeomorphisms of metric products," Proceedings of the American Mathematical Society, vol. 103, no. 4, pp. 1293-1298, 1988.

[3] R. Dubey and A. Vyas, "Wavelets and the complete invariance property," Matematichki Vesnik, vol. 62, no. 2, pp. 183-188, 2010.

[4] P. Wojtaszczyk, A Mathematical Introduction to Wavelets, Cambridge University Press, 1997.

[5] M. Bownik, "A characterization of affine dual frames in $L^{2}\left(\mathbb{R}^{n}\right)$," Applied and Computational Harmonic Analysis, vol. 8, no. 2, pp. 203-221, 2000.
[6] M. Bownik, Z. Rzeszotnik, and D. Speegle, "A characterization of dimension functions of wavelets," Applied and Computational Harmonic Analysis, vol. 10, no. 1, pp. 71-92, 2001.

[7] X. Dai, D. R. Larson, and D. M. Speegle, "Wavelet sets in $\mathrm{R}^{n}$," The Journal of Fourier Analysis and Applications, vol. 3, no. 4, pp. 451-456, 1997.

[8] D. Han and D. R. Larson, "Frames, bases and group representations," Memoirs of the American Mathematical Society, vol. 147, no. $697,2000$.

[9] Z. Li and X. Shi, "On Parseval super frame wavelets," Applied Mathematics-A Journal of Chinese Universities, vol. 27, no. 2, pp. 192-204, 2012.

[10] H. Robbins, "Some complements to Brouwer's fixed point theorem," Israel Journal of Mathematics, vol. 5, pp. 225-226, 1967. 


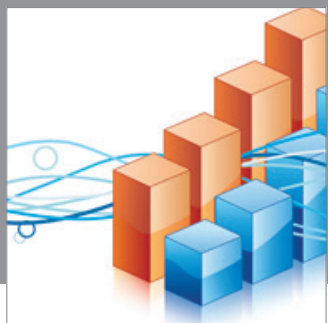

Advances in

Operations Research

mansans

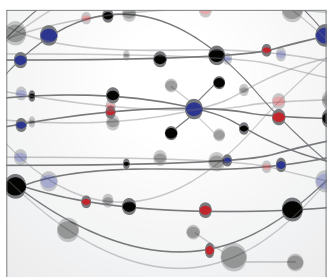

The Scientific World Journal
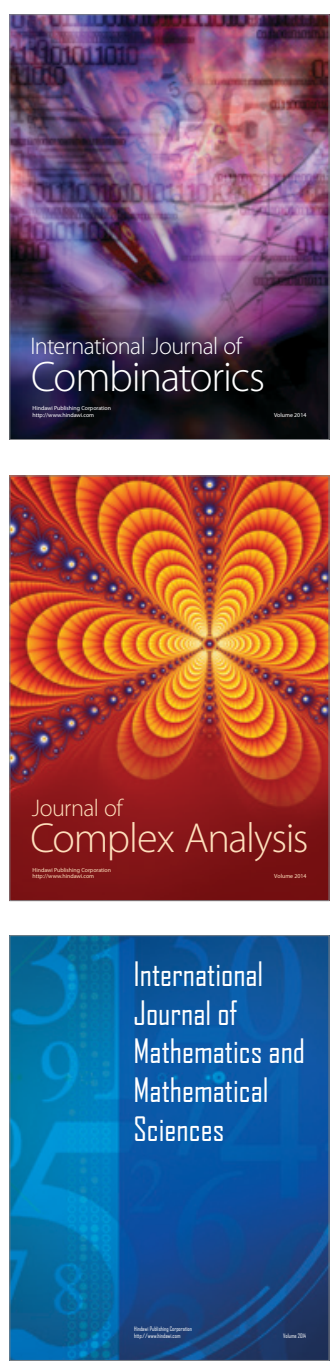
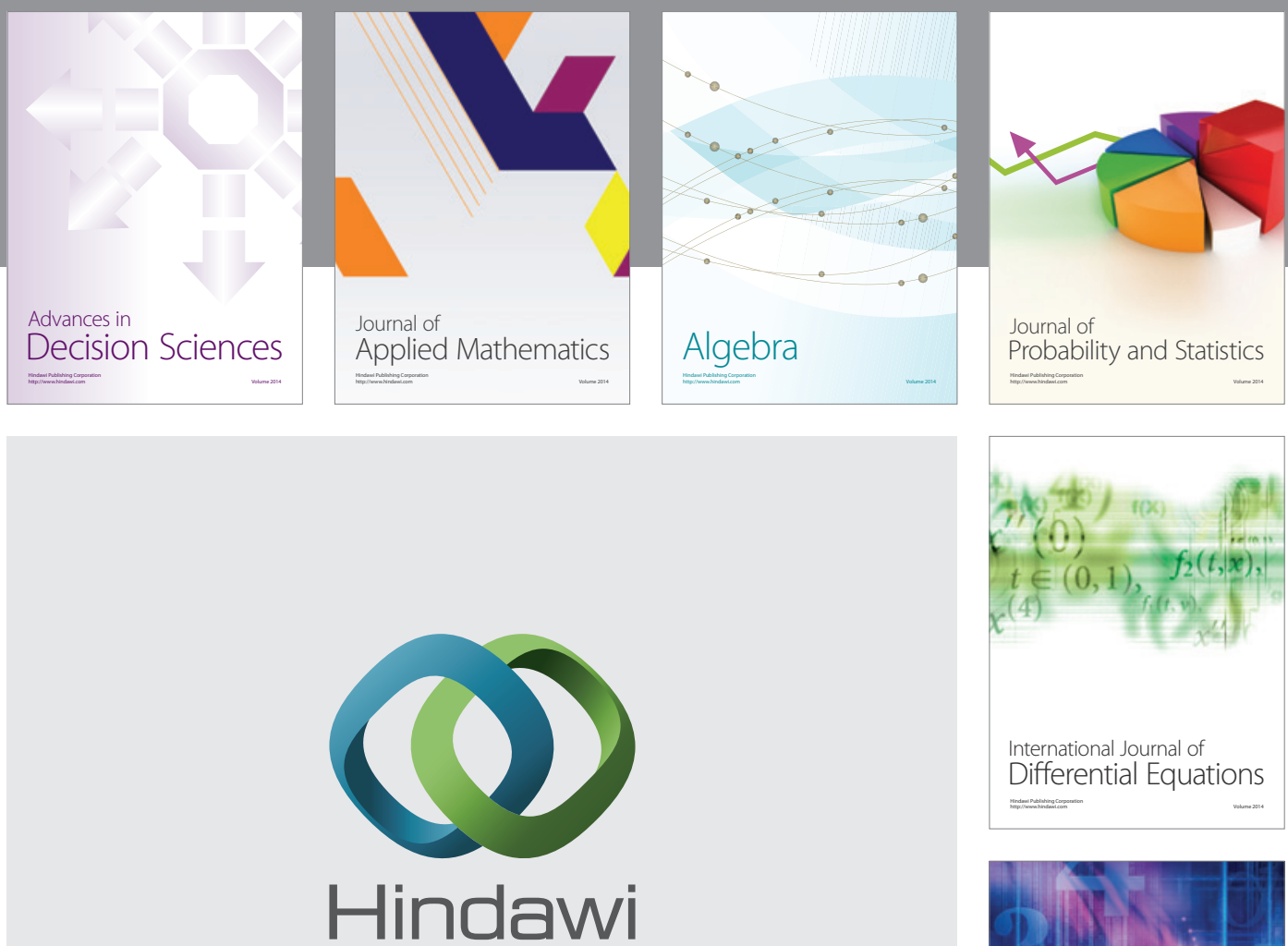

Submit your manuscripts at http://www.hindawi.com
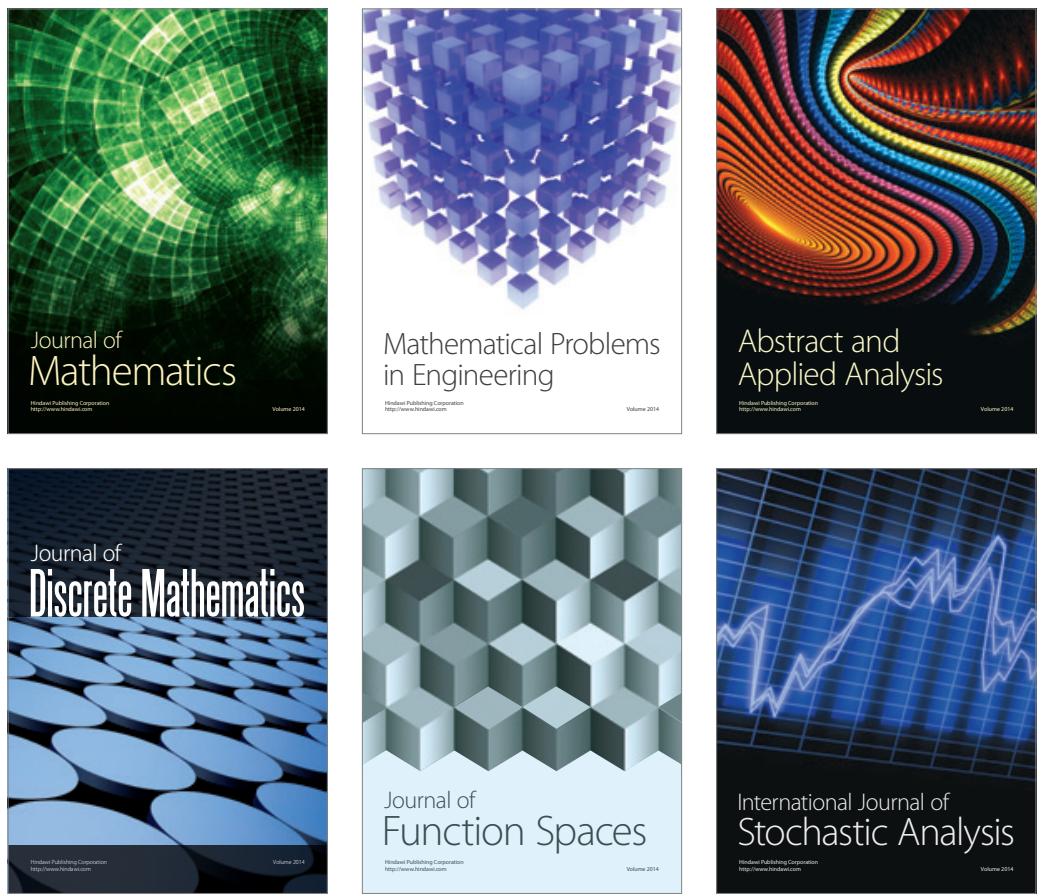

Journal of

Function Spaces

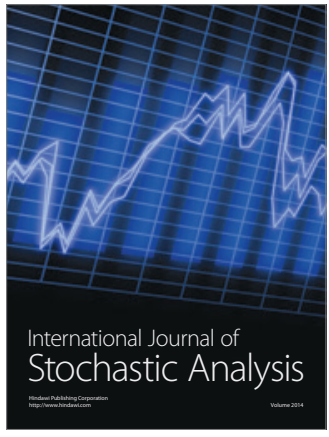

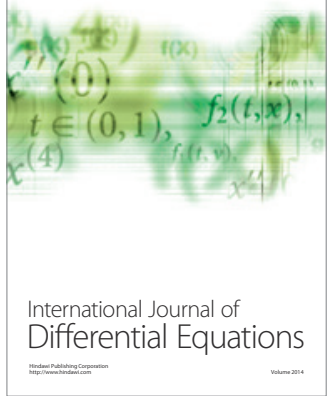
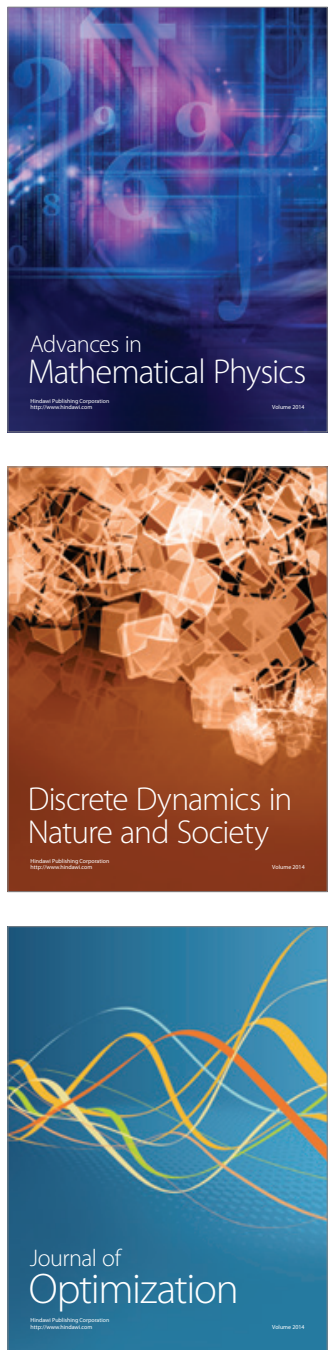